\title{
Efectos en el Sistema Suelo-Planta Después de Tres Años de Aplicación de Purín de Cerdo como Fertilizante en un Cultivo de Brócoli (Brassica oleracea L. $)^{1}$
}

\author{
Miriam Llona $\mathrm{C}^{2}$. y Angel Faz $\mathrm{C}^{3}$ \\ ${ }^{2}$ Escuela de Ciencias Biológicas y Químicas. Facultad de Recursos Naturales. Universi- \\ dad Católica de Temuco. Montt 56, Temuco-Chile, casilla 15-D. \\ Correspondencia: $\underline{\text { mllona@uct.cl }}$ \\ ${ }^{3}$ Área de Edafología y Química Agrícola. Departamento de Ciencia y Tecnología Agraria. \\ Universidad Politécnica de Cartagena. Paseo Alfonso XIII, 52. 30203 Cartagena. \\ Murcia. España.
}

\section{Effects in the soil-plant system after three years of application of pig slurry as fertilizer in a broccoli (Brassica oleracea L.) crop}

Key words: Pig slurry, soil physical-chemical characteristics, broccoli, fertilizer

\begin{abstract}
This paper is aimed to determine the dose of correct application of pig slurry as fertilizer minimizing its polluting effects. It was experienced with a horticultural crop, broccoli, representative of the study area. The experience lasted three years and was carried out in four plots $\left(630 \mathrm{~m}^{2}\right)$ with different applications of pig slurry: the treatments were: without slurry and $4,86 \mathrm{~L} / \mathrm{m}^{2} ; 11,05 \mathrm{~L} / \mathrm{m}^{2}$ and $14,86 \mathrm{~L} / \mathrm{m}^{2}$ of slurry application namely: control, B, C and $\mathrm{D}$, respectively. After the experience, the soil presented important increments in $\mathrm{N}, \mathrm{P}, \mathrm{K}$ and organic carbon, especially after successive applications and high doses. It was also observed an slight increase of the $\mathrm{pH}$ and a decreasing of electric conductivity; the oligoelements increased without exceeding the limits ruled by the legislation. The plant tissue increased in N, P and K after successive slurry applications. The same happened with the oligoelements and $\mathrm{NO}_{3}^{-}$; however, these values were in the normal range, not surpassing the limit imposed by law. The broccoli production increased when increased the slurry doses in successive applications, surpassing the stocking of production of the Valley of the Guadalentín.
\end{abstract}

Palabras claves: Purín de cerdo, características físico-químicas suelo, brócoli, fertilizante.

\footnotetext{
${ }^{1}$ Basado en Tesis Doctoral Universidad Politécnica de Cartagena Murcia-España, departamento de Ciencia y Tecnología Agraria.
} 


\section{RESUMEN}

Este trabajo tiene como objetivo determinar la dosis de aplicación correcta de purines de cerdo como fertilizante minimizando sus efectos contaminantes. Para ello se experimentó con un cultivo hortícola de riego representativo de la zona de estudio. La experiencia, que duró 3 años, cultivando brócoli, se realizó en 4 parcelas $\left(630 \mathrm{~m}^{2}\right)$ con distintas aplicaciones de purín de cerdo: sin purín y tres dosis de $4,86 \mathrm{~L} / \mathrm{m}^{2}, 11,05 \mathrm{~L} / \mathrm{m}^{2}$ y $14,86 \mathrm{~L} / \mathrm{m}^{2}$, parcelas control, B, C y D respectivamente. Tras la experiencia, el suelo presenta incrementos importantes en N, P, K y carbono orgánico, especialmente después de sucesivas aplicaciones y en dosis altas, además, se observa un ligero aumento del $\mathrm{pH}$ y un descenso de la conductividad eléctrica; los oligoelementos experimentan un aumento sin exceder los límites establecidos por la legislación. La planta presenta aumentos en N, P y K tras sucesivas aplicaciones de purín y en dosis altas, al igual que ocurre con el contenido en oligoelementos y $\mathrm{NO}_{3} ;$; sin embargo, estos valores se encuentran dentro de rangos normales, no sobrepasando lo establecido por la legislación. La producción aumenta al incrementar la dosis de purín y en aplicaciones sucesivas, sobrepasando la media de producción del Valle del Guadalentín.

\section{INTRODUCCIÓN}

El hecho de que los purines de cerdo actualmente constituyan un problema medio ambiental grave se debe principalmente al cambio en el sistema de explotación ganadera, ligado a su intensificación. Entre los años 60 y 90, hubo en España una reducción de las explotaciones de porcino con tierra del $75 \%$ (Coll, 1993). Esta tendencia a un sistema productivo de tipo intensivo, con explotaciones sin tierra, alta mecanización, alimentación a base de piensos compuestos, etc., lleva consigo una serie de consecuencias, la más importante consiste en que los purines producidos no tienen un área territorial donde pueden ser aplicados o bien vertidos; además, la especialización de algunas zonas, que hacen de la ganadería porcina una producción casi exclusiva, aumenta esta problemática.

Por otra parte, el suelo, como elemento dinámico, permite, entre otras funciones, filtrar, amortiguar, degradar, inmovilizar y reducir la toxicidad de materiales orgánicos e inorgánicos, incluyendo subproductos urbanos e industriales y depósitos atmosféricos (Seybold et al., 1998). Su capacidad para la biodegradación de residuos y el hecho de que los elementos contaminantes per- manezcan en el suelo durante más tiempo sin que se observen a corto plazo efectos nocivos, comparado con el agua o la atmósfera, hacen de este el elemento un recurso más "resistente". Sin embargo esta capacidad es limitada, provocando en muchos casos la degradación del suelo y por consiguiente la disminución de su capacidad actual o futura para generar, en términos de calidad y cantidad, bienes o servicios.

Diversos estudios coinciden en afirmar que el método más efectivo y económico de afrontar la problemática de la acumulación de los desechos de tipo orgánico procedentes de la ganadería intensiva, es a través de su aplicación en el suelo (Westernam y Bicudo, 2005; Schröder et al., 2004). La aplicación racional y controlada de los purines de cerdo al suelo presenta un gran interés desde el punto de vista de la fertilización, en suelos que han sido durante siglos utilizados para la agricultura y que, por lo tanto, están empobrecidos respecto a sus cualidades nutritivas. También es de resaltar la importancia que tiene el hecho de reemplazar la fertilización mineral por la de tipo orgánica, lo que lleva a minimizar los costes de producción, a la vez que ayuda a mejorar las condiciones medioambientales (Schröder, 2005). No se debe olvidar que la 
fertilización mineral aplicada en forma no controlada también puede ocasionar serios problemas de contaminación y degradación del suelo (Goulding, 2000).

La producción de ganado porcino en España sobrepasa los 22 millones de cabezas. Esta producción se concentra mayoritariamente en 10 Comunidades Autónomas, siendo la Región de Murcia la quinta con cerca de los 2 millones de cabezas y segunda después de Cataluña en número de cabezas por superficie. Dentro de la Región de Murcia el Municipio de Lorca es el que posee un mayor número de explotaciones, con 2657 (43\%) llegando a producir cerca de los 4 millones de $\mathrm{m}^{3}$ de purín al año (MAPYA, 2003).
El objetivo de este trabajo es evaluar la evolución en los parámetros químicos del suelo tras la aplicación de diferente dosis de purines de cerdo como fertilizante en un cultivo de brócoli.

\section{MATERIALESYMÉTODOS}

Este estudio se ha llevó a cabo en el Centro Integrado de Formación y Experiencias Agrarias de Lorca (CIFEA), perteneciente a la Consejería de Agricultura y Agua de la Comunidad Autónoma de la Región de Murcia, en el Valle del Guadalentín Sudeste de España (Figura 1).

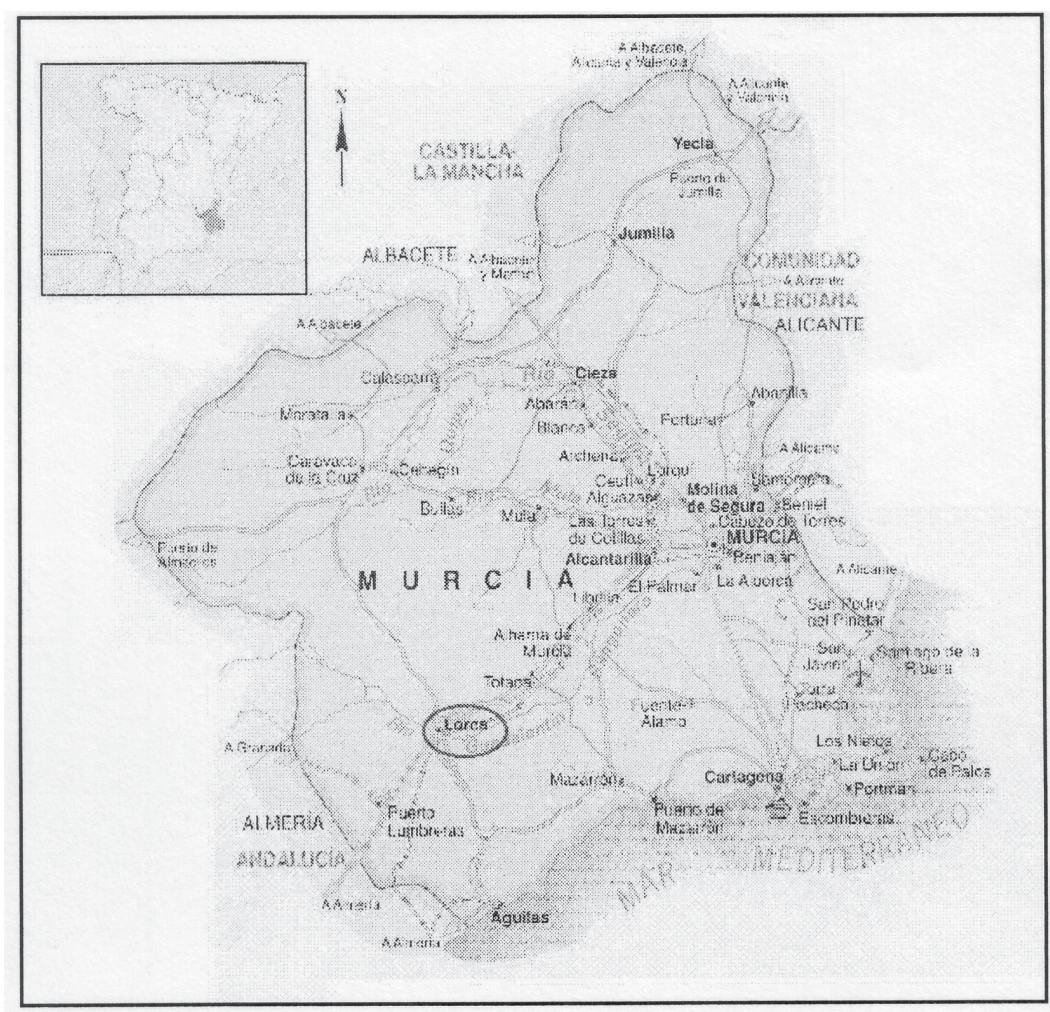

Figura 1. Localización área de estudio

Figure 1. Study locatoion 
La precipitación media anual alcanza valores que oscilan entre 200 a $300 \mathrm{~mm}$, mientras que la temperatura media anual, relativamente alta, fue de $18^{\circ} \mathrm{C}$. En invierno las temperaturas media es de $6{ }^{\circ} \mathrm{C}$ y en verano de $36^{\circ} \mathrm{C}$, razón por la cual existe una elevada evapotranspiración, por lo que se puede considerar a esta zona bajo un régimen de humedad del suelo de tipo arídico (EEUU, 2003). Los suelos de esta zona son Fluvisoles calcáricos (FAO-ISRIC-ISSS, 1998).

En relación al análisis general del perfil, se observa un descenso en el contenido de nitrógeno y carbonato cálcico a medida que se avanza en profundidad, sin embargo, las concentraciones de carbono orgánico, capacidad de intercambio catiónica y conductividad eléctrica presentan valores más altos en el horizonte $\mathrm{C} 1$. El pH tiende a aumentar en los horizontes más profundos. En los elementos asimilables se distinguen, en general, incrementos en profundidad, excepto en las concentraciones de K y P. Por otra parte, los elementos solubles se concentran especialmente en el horizonte $\mathrm{C} 1$. Respecto a la granulometría, el horizonte $\mathrm{C} 1$ es el que presenta un mayor porcentaje de arcillas, sobrepasando el $50 \%$, en cambio, el horizonte Ap2 es el que presenta el menor porcentaje de partículas inferiores a $2 \mu \mathrm{m}$ (Cuadro 1).

La experiencia, que duró 3 años, cultivando brócoli, se realizó en 4 parcelas $\left(630 \mathrm{~m}^{2}\right)$ con distintas aplicaciones de purín de cerdo: $\sin$ purín y tres dosis de $4,86 \mathrm{~L} / \mathrm{m}^{2}$, $11,05 \mathrm{~L} / \mathrm{m}^{2}$ y $14,86 \mathrm{~L} / \mathrm{m}^{2}$, parcelas control, B, $\mathrm{C}$ y D respectivamente. Las parcelas $\mathrm{B}, \mathrm{C}$ y $\mathrm{D}$ se subdividieron en 3 subparcelas $\mathrm{B}_{1}, \mathrm{~B}_{2}$, $\mathrm{B}_{3}, \mathrm{C}_{1}, \mathrm{C}_{2}, \mathrm{C}_{3}$, etc, de $21 \mathrm{~m} \times 10 \mathrm{~m}$, para efectos estadísticos; la parcela control (A), en cambio, no se subdividió, existiendo una única parcela de $21 \mathrm{~m}$ x $10 \mathrm{~m}$. Para observar el efecto residual y acumulativo a partir del segundo año se subdividieron las parcelas

Cuadro 1. Características físico-químicas del purín de cerdo aplicado al cultivo de brócoli. media de las 3 dosis; $4,86 \mathrm{~L} / \mathrm{m}^{2}, 11,05 \mathrm{~L} / \mathrm{m}^{2}$ y $14,86 \mathrm{~L} / \mathrm{m}^{2}$

\begin{tabular}{cc}
\hline $\mathrm{pH}$ & 8 \\
Conductividad Eléctrica dS m & 12 \\
Carbono Orgánico $\mathrm{g} \mathrm{L}^{-1}$ & 10 \\
Nitrógeno Total $\mathrm{g} \mathrm{L}^{-1}$ & 2 \\
Nitrógeno Amoniacal g L & 1,6 \\
$\mathrm{Na} \mathrm{mg} \mathrm{L}^{-1}$ & 588 \\
$\mathrm{~K} \mathrm{mg} \mathrm{L}^{-1}$ & 1668 \\
$\mathrm{PO}_{4}^{-3} \mathrm{mg} \mathrm{L}^{-1}$ & 952 \\
$\mathrm{Humedad} \%$ & 97 \\
\hline
\end{tabular}


en dos parcelas de $10 \mathrm{~m}$ x $10 \mathrm{~m}$ quedando una como efecto residual (no se vuelve a aplicar purín) y otra de efecto acumulativo (se sigue aplicando purín) (Figura 2).

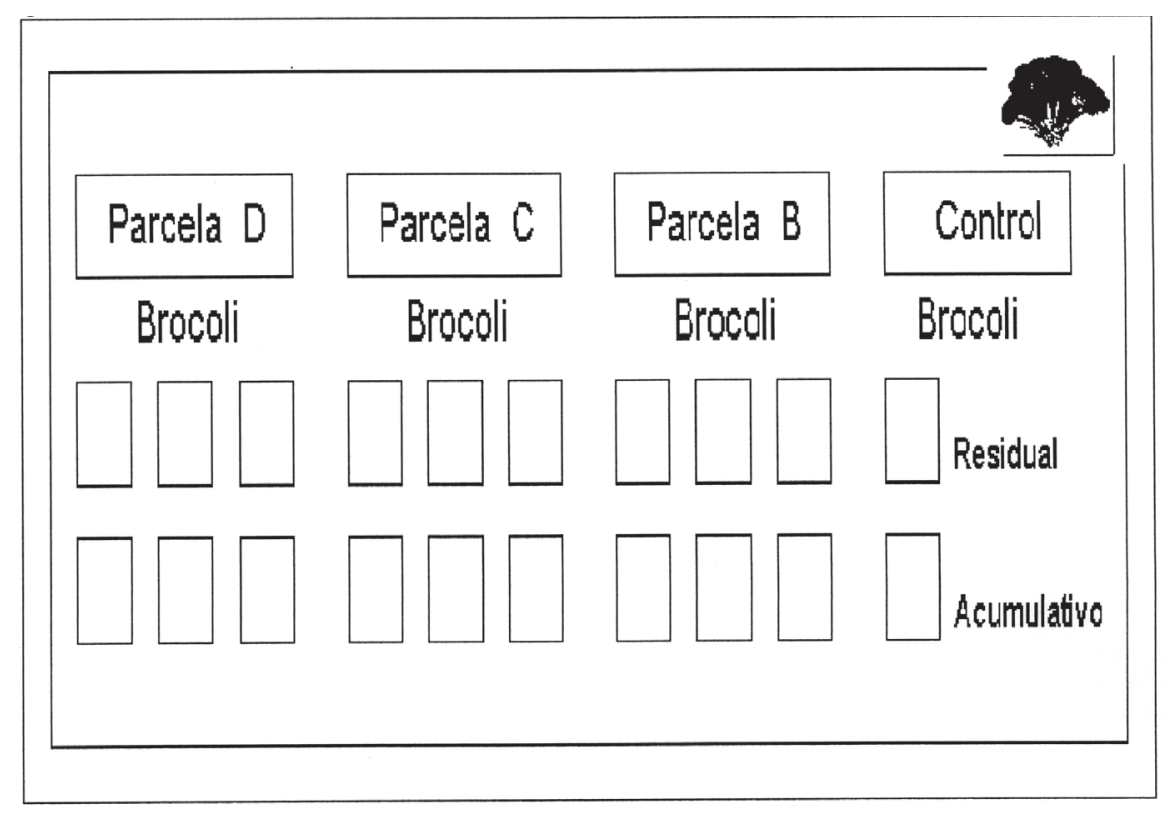

Figura 2. Esquema parcelas de estudio

Figure 2. Experimental layout

Las muestras de purín se extrajeron por cada aplicación. Las muestras de suelo se extrajeron dos veces al año por cultivo, antes del inicio del cultivo (SP) y al terminar la cosecha (CP). En cada parcela (control, B, C y D), con sus respectivas repeticiones. $\mathrm{Se}$ extrajeron muestras de la superficie del suelo $(0-30 \mathrm{~cm})$ y en profundidad $(30-60 \mathrm{~cm})$. Los métodos usados en purín son los recomendados para fertilizantes en MAPYA (1998)

Previamente a la realización de los análisis de laboratorio, las muestras de suelo se secaron al aire y posteriormente se separaron mediante un tamiz de $2 \mathrm{~mm}$, utilizando la proporción menor de este diámetro para los análisis. Además parte de cada muestra se molió para la determinación de algunos constituyentes. Las variables analizadas furon: pH (Peech, 1965), conductividad eléctrica del extracto de saturación (CE) (Bower y Wilcox, 1965), carbono orgánico total (C) (Anne,1945), modificado por Duchaufour (1970), nitrógeno total (N) (Duchaufour, 1970), potasio de cambio o asimilables (K) (Pratt, 1965), fósforo asimilable (P) (Watanabe y Olsen,1965), oligoelementos bioasimilables $(\mathrm{Cu}, \mathrm{Zn})$ (Lindsay y Norwell, 1969). Los análisis de planta se realizaron en hoja (Madrid et. al., 1996). Se realizó el análisis de la varianza utilizando ANOVA de una sola vía. 


\section{RESULTADOS Y DISCUSIÓN}

\section{Suelo}

El pH presenta un aumento a medida que se incrementa la dosis de purín, tanto en superficie como en profundidad; no se observó gran variación entre el efecto residual y acumulativo (Figura 3 y 4 ).

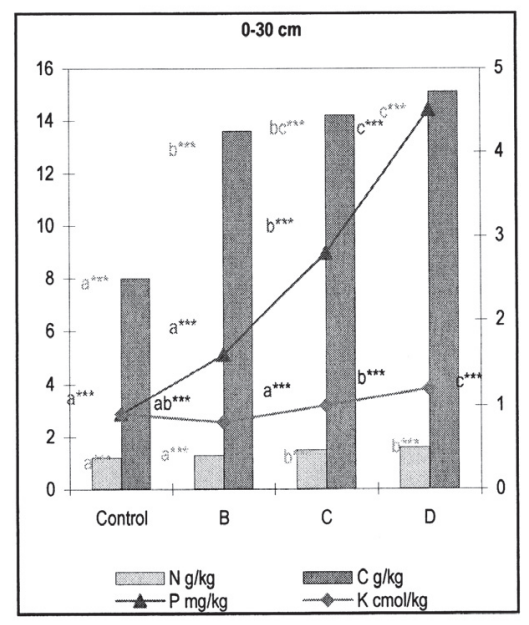

Figura 3. Características del suelo $(0-30 \mathrm{~cm})$ cultivado con brócoli, SP: antes de la aplicación del purín, CP: después de la aplicación. Parcela control y parcelas B, C y D enmendadas con purín de cerdo en las siguientes dosis: $4,86 \mathrm{~L} / \mathrm{m}^{2}, 11,05 \mathrm{~L} / \mathrm{m}^{2}$ y $14,86 \mathrm{~L} / \mathrm{m}^{2}$. $\mathrm{N}$ : Nitrógeno total; C: Carbono orgánico total; P: Fósforo asimilable; K: potasio de cambio o asimilable. *, $* *, * * *$ : significativo al nivel de probabilidad 0,05 ; 0,$01 ; 0,001$; respectivamente. ns: no significativo al nivel 0,05 . Valores dentro de la misma columna seguidos de la misma letra no son significativamente distintos según el test $\mathrm{LSD}(\mathrm{P}=0,05)$.

Figure 3. Soil characteristic $(0-30 \mathrm{~cm})$ cropped with broccoli, SP: before pig slurry application, CP: after application. Control plot and plots B, C and D, amended with pig slurry in the following doses: 4.86 $\mathrm{L} / \mathrm{m}^{2}, 11.05 \mathrm{~L} / \mathrm{m}^{2}$ y $14.86 \mathrm{~L} / \mathrm{m}^{2}$. N: total nitrogen; C: Organic carbon; $\mathrm{P}$ : available phosphorus; $\mathrm{K}$ : available potassium. *, **,***: significant at $0.05 ; 0.01 ; 0.001$ probability level, respectively. ns: not significant at 0.05 level. Values within the same column followed by the same letter are not significant, $\operatorname{LSD}(\mathrm{P}=0.05)$.

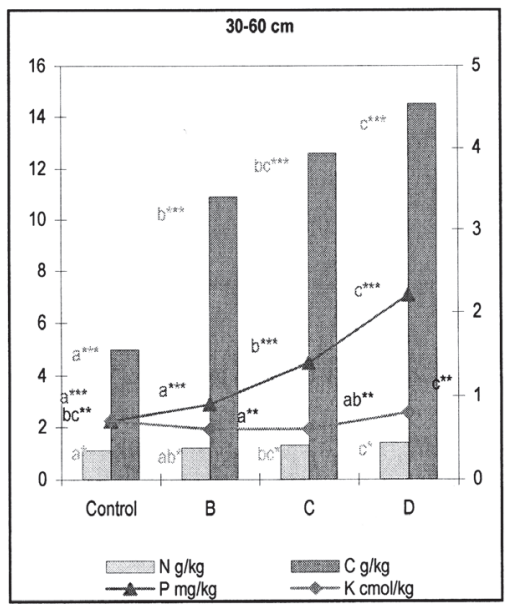

Figura 4. Características del suelo $(30-60 \mathrm{~cm})$ cultivado con brócoli. Ver leyenda en Figura 3.

Figure 4. Soil characteristic $(30-60 \mathrm{~cm})$ cropped with broccoli. For descriptive legend see Figure 3 .

Existe una clara relación entre las diferentes dosis, a partir del segundo año de la aplicación de purín. Este incremento se pudo deber, por una parte, al aporte de macroelementos como N, P y K (Erich, et al., 2002; Martinez y Hao, 1996), y por la otra a que el $\mathrm{Na}$ contenido en el purín de cerdo (Cuadro 2) e incorporado al suelo se puede considerar como un elemento activo en lo que se refiere a la alcalinidad. De otro modo, la absorción de $\mathrm{NO}_{3}^{-}$por la planta, y la desnitrificación del ion nítrico a $\mathrm{NH}_{4}^{+}$(Xu et al., 1998; Simek et al., 2000), puede también conllevar un ascenso en el $\mathrm{pH}$ del suelo.

La conductividad eléctrica tiende a disminuir, especialmente después de la aplicación de purín, tanto en superficie (Figuras 3) como en profundidad, correspondiendo a este último los mayores rangos de diferencia (Figura 4 ); esto se debe a la influencia que ejerce la humedad contendida en el suelo en la conductividad eléctrica, debido a las sales disueltas en la solución. A pesar de que la mayoría de los autores obtienen como resultado un aumento de la conductividad eléctrica al incorporar purín de cerdo (Ye et al., 1999), otros como Plaza (2002) obtienen incrementos menos acusados en los últimos años de experimentación. 
Murcia (2003a) y Murcia (2003b) observaron descensos en la conductividad eléctrica tras la aplicación de purín en cultivo de brócoli, aplicación que se llevó a cabo de igual forma que en el presente estudio. Esta disminución se presentó en superficie elevándose la conductividad eléctrica en profundidad (30-60 cm). Lo que ratifica la hipótesis planteada. Sin embargo, debido a que en este estudio el descenso también se presenta en profundidad, es posible que esta lixiviación se produzca hasta el horizonte $\mathrm{C} 1$, situado por debajo de los $32 \mathrm{~cm}$, cuya clase textural es arcillo limosa, con más de $50 \%$ en el contenido de arcilla; una conductividad eléctrica de $7 \mathrm{dS} \mathrm{m}^{-1}$ (Cuadro 1) y una importante concentración de sales solubles, especialmente $\mathrm{Cl}^{-}$y $\mathrm{SO}_{4}^{-}$de origen geoquímico fundamentalmente.

El contenido de carbono orgánico aumenta notoriamente tras la aplicación de purín. Es de destacar el ascenso de este constituyente a medida que se incrementa la dosis de purín, tanto en efecto residual como acumulativo (Figura 5 y 6 ).

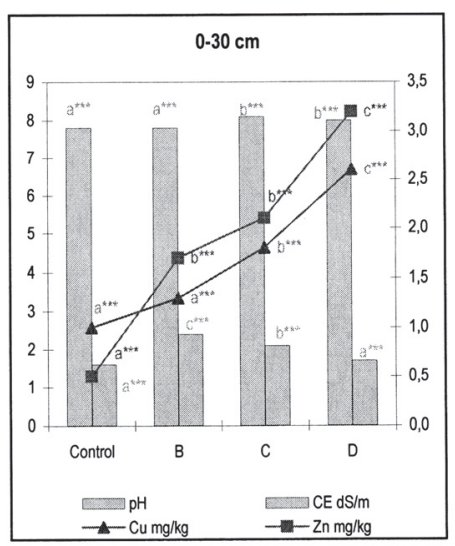

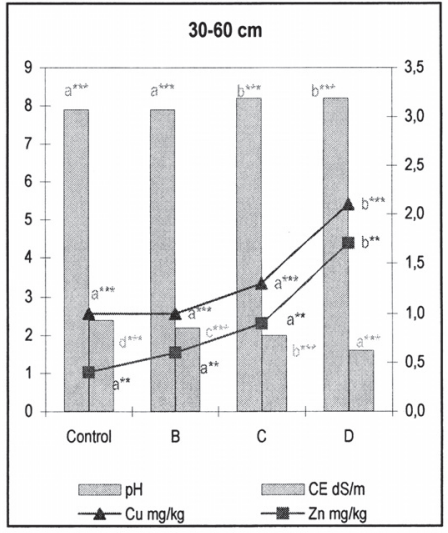

Figura 6. Características del suelo $(30-60 \mathrm{~cm})$ cultivado con brócoli. Ver leyenda en Figura 5.

Figure 6. Soil characteristic $(30-60 \mathrm{~cm})$ cropped with broccoli. For descriptive legend see Figure 5.

En general, los valores de carbono orgánico son mayores en superficie. A pesar de que el contenido en carbono orgánico en purín es bajo, comparado con otros tipos de estiércoles, especialmente aquellos que contienen un menor porcentaje de humedad (Adegbidi et al., 2003), los resultados obtenidos muestran claramente un aumento del carbono orgánico en suelo (Rochette et al., 2000a).

Sin duda, el nitrógeno es uno de los elementos más abundantes en el purín (Cuadro 2) y, por tanto, actúa más rápidamente en el suelo, de modo que los efectos a la hora de aplicarlo se evidencian a corto plazo (Goulding et al., 2000; Rochette et al., 2000b; Sørensen y Amato, 2002). Se observa una correlación entre las diferentes dosis de purín y el contenido de nitrógeno en

Figura 5. Características del suelo $(0-30 \mathrm{~cm})$ cultivado con brócoli, $\mathrm{SP}$ : antes de la aplicación del purín, $\mathrm{CP}$ : después de la aplicación. Parcela control y parcelas B, C y D enmendadas con purín de cerdo en las siguientes dosis: 4,86 L/m², 11,05 L/m² y 14,86 L/m².pH medido en $\mathrm{H}_{2} \mathrm{O}$; CE: Conductividad Eléctrica del extracto de saturación; $\mathrm{Cu}$ : Cobre bioasimilable; Zn: Zinc bioasimilable. *, **, ***: significativo al nivel de probabilidad 0,$05 ; 0,01 ; 0,001$; respectivamente. ns: no significativo al nivel 0,05 . Valores dentro de la misma columna seguidos de la misma letra no son significativamente distintos según el test $\operatorname{LSD}(\mathrm{P}=0,05)$.

Figure 5. Soil characteristic $(0-30 \mathrm{~cm})$ cropped with broccoli, SP: before pig slurry application, CP: after application. Control plot and plots B, C and D, amended with pig slurry in the following doses: $4.86 \mathrm{~L} / \mathrm{m}^{2}$, $11.05 \mathrm{~L} / \mathrm{m}^{2}$ y $14.86 \mathrm{~L} / \mathrm{m}^{2}$. water $\mathrm{pH}, \mathrm{CE}$ : electrical conductivity; $\mathrm{Cu}$ : available copper; $\mathrm{Zn}$ : available zinc *, $* *$, ***: significant at $0.05 ; 0.01 ; 0.001$ probability level, respectively. ns: not significant at 0.05 level. Values within the same column followed by the same letter are not significant, $\operatorname{LSD}(\mathrm{P}=0.05)$. 
suelo, siendo, en general, mayor en las dosis más altas, tanto en efecto residual como acumulativo (Figuras 5 y 6), desde el primero, tras la aplicación de purín, hasta el último año de experimentación.

El contenido de $\mathrm{K}$, al igual que el $\mathrm{N}$, experimenta un importante aumento, evidenciado por una respuesta rápida, por parte del suelo, debido a su abundancia en el purín (Cuadro 2) (Piccinini y Bortone, 1991; Sánchez, 2001). Existe correlación entre las diferentes dosis y el contenido en $\mathrm{K}$, desde el primer año de experimentación después de la aplicación de purín, tanto en superficie como en profundidad; continuando esta tendencia en los dos años siguientes (Figuras 5 y 6 ).

Existe un aumento importante en el contenido de $\mathrm{P}$ en las parcelas con las dosis media y alta, incremento que se evidencia ya en el primer año después de la incorporación de purín, por la importante concentración de este elemento en el purín (Cuadro 2) (Sánchez, 2001; Plaza 2002). Se observan notorias diferencias entre efecto residual y acumulativo. La correlación existente entre las diferentes dosis de purín y el valor de cada variable se observa desde el primer año después de la aplicación de purín: en todos los casos, el valor de $\mathrm{P}$ aumenta a medida que se incrementa la dosis de purín tanto en superficie como en las muestras de profundidad (Figuras 5 y 6), (Papini et al., 1994; Anderson y Wu, 2001)

En general, los valores obtenidos en $\mathrm{Cu}$ y $\mathrm{Zn}$ bioasimilables tienen una tendencia a subir a medida que se incorpora una mayor cantidad de purín año tras año (Figuras 3 y 4) (L'Herroux et al., 1997; Martinez y Peu, 2000); a pesar del ascenso del pH provocando inmovilidad en estos metales. Sin embargo, el contenido en carbono orgánico, ejerce una importante influencia en la movilidad de los metales en suelos con $\mathrm{pH}$ altos (Ashworth y Alloway, 2004; Walker et al., 2003).

\section{Planta}

$\mathrm{N}$, P y K totales presentan las concentraciones mayores en las plantas en el primer año de experimentación, bajando luego en el segundo y tercer año. Existen diferencias entre el efecto residual y acumulativo siendo este último el que presenta las concentraciones mayores, estas siempre en las dosis más altas (Figura 7); aún así, en el contenido de $\mathrm{P}$ y $\mathrm{N}$ total se observan diferencias significativas en mayor proporción que en el contenido de $\mathrm{K}$ en planta. Las concentraciones de estos tres elementos están dentro de rangos normales para planta madura (Azcón-Bieto y Talón, 2000).

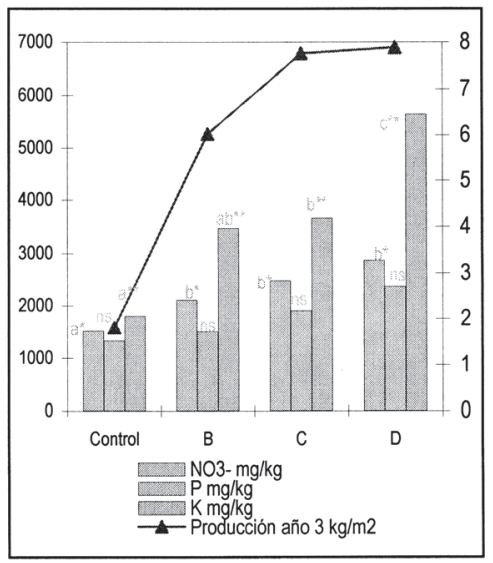

Figura 7.Evolución de las características nutricionales de la planta (hoja) en cultivo de brócoli. SP: antes de la aplicación del purín, CP: después de la aplicación. Parcela control y parcelas B, C y D enmendadas con purín de cerdo en las siguientes dosis: $4,86 \mathrm{~L} / \mathrm{m}^{2}$, $11,05 \mathrm{~L} / \mathrm{m}^{2}$ y $14,86 \mathrm{~L} / \mathrm{m}^{2}$. Producción $\left(\mathrm{kg} / \mathrm{m}^{2}\right)$. * **, ***: significativo al nivel de probabilidad 0,$05 ; 0,01$; 0,001 ; respectivamente. ns: no significativo al nivel 0,05 . Valores dentro de la misma columna seguidos de la misma letra no son significativamente distintos según el test $\mathrm{LSD}(\mathrm{P}=0,05)$.

Figure 7. Characteristic of plant (leaf) nutrient evolution in the brócoli crop. SP: before pig slurry application, CP: after application. Control plot and plots $\mathrm{B}, \mathrm{C}$ and $\mathrm{D}$, amended with pig slurry in the following doses: $4.86 \mathrm{~L} / \mathrm{m}^{2}, 11.05 \mathrm{~L} / \mathrm{m}^{2}$ y $14.86 \mathrm{~L} / \mathrm{m}^{2}$. Broccoli production $\left(\mathrm{kg} / \mathrm{m}^{2}\right)$. *,**,***: significant at $0.05 ; 0.01 ; 0.001$ probability level, respectively. ns: not significant at 0.05 level. Values within the same column followed by the same letter are not significant, $\operatorname{LSD}(\mathrm{P}=0.05)$. 
Se presentó un incremento de la concentración de nitratos en planta correspondiendo los valores más altos a dosis altas y en sucesivas aplicaciones de purín (Figura 7). Sin embargo, estos valores no sobrepasaron lo estipulado por la ley que establece como máximo $4500 \mathrm{mg} \mathrm{kg}^{-1}$ de nitratos en planta fresca (Reglamento (CE) No 563/2002). Sin embargo, es uno de los aspecto a tomar en cuenta en relación a los riesgos respecto a la excesiva aplicación de purín en cultivos hortícolas.

Se observa un claro aumento de la producción de brócoli en sucesivas aplicaciones de purín; este incremento se produce a mayores dosis superando al primer año de experimentación y a la media de la zona de estudio (Figura 7).

\section{CONCLUSIONES}

El suelo presenta incrementos importantes en N, P y K, especialmente después de sucesivas aplicaciones y en dosis altas, por la abundante concentración en el purín de estos elementos. También se observa un ligero aumento del $\mathrm{pH}$ y un descenso de la conductividad eléctrica.

El contenido de carbono orgánico aumenta notoriamente tras la aplicación de purín. Los oligoelementos experimentan un aumento sin exceder los límites establecidos por la legislación.

La planta presenta aumentos en N, P y $\mathrm{K}$ tras sucesivas aplicaciones de purín y en dosis altas, al igual que ocurre con el contenido en oligoelementos y $\mathrm{NO}_{3}^{-}$; sin embargo, estos valores se encuentran dentro de rangos normales, no sobrepasando lo establecido por la legislación. La producción aumenta al incrementar la dosis de purín y en aplicaciones sucesivas, sobrepasando la media de producción del Valle del Guadalentín.

\section{AGRADECIMIENTOS}

Este proyecto fue financiado por la Consejería de Agricultura y Agua de España y la Federación de Cooperativas Agrarias de Murcia-España (FECOAM). Agradezco a CONICYT por el otorgamiento de la beca "Gobierno de Chile-BID” que permitió la financiación de mis estudios de doctorado.

\section{BIBLIOGRAFÍA}

ADEGBIDI, H., BRIGGS, R., VOLK, T., WHITE, E. y ABRAHAMSON, L. 2003. Effect of organic amendments and slowrelease nitrogen fertilizer on willow biomass production and soil chemical characteristics. Biomass an Bioenergy. 25: 389-398.

ANDERSON, R. y WU, Y. 2001. Phosphorus quantity-intensity relationships and agronomic measures of $\mathrm{P}$ in surface layers of soil from a long-term slurry experiment. Chemosphere. 42: 161-170.

ANNE, A. 1945. Ann., Agro. 2: 161-172.

ASHWORTH, D.J. y ALLOWAY, B.J. 2004. Soil mobility of sewage sludge-derived dissolved organic matter, copper, nickel and zinc. Environmental Pollution. 127: 137-144.

AZCÓN-BIETO, J. y TALÓN, M. 2000. Fundamentos de fisiología vegetal. McGraw-Hill, Madrid. $522 \mathrm{p}$.

BOWER, C. A. y WILCOX, L. V. 1965. Soluble salts. In C. A. Black (ed.). Methods of Soils Analysis. American Society of Agronomy. Madison, Wisconsin, U.S.A. 2, 933-940.

BURTON, C.H y TURNER, C. 2003. Manure Management, treatment strategies for sustainable agriculture. Silsoe Research Institute, Silsoe. UK. 490 p.

COLL, D. 1993. La intensificación ganadera como proceso de producción de residuos. En Residuos Ganaderos. Jornadas Técnicas organizadas por Fundación "La Caixa" y editorial AEDOS. 5-15. 
DUCHAUFOUR, P h. 1970 . P r é c is de Pédologie. Masson. Paris. $481 \mathrm{p}$.

ERICH, M. S., FITZGERALD, C.B. y PORTER, G.A. 2002. The effects of organic amendments on phosphorus chemistry in potato cropping system. Agriculture, Ecosystems and Environment. 88:79-88.

FAO.- ISRIC - ISSS 1998. World Reference Base for Soil Resources (Ed), Rome. 84 p.

GOULDING, K., POULTON, P., WEBSTER, C. y HOWE, M. 2000. Nitrate leaching from the Broadbalk Wheat experiment, Rothamstes, UK, as influenced by fertilizer and manure inputs and the weather. Soil Use and Management. 16: 244-250.

L' HERROUX, L., LE ROUX, S., APPRIOU, P. y MARTINEZ, J. 1997. Behaviour of metals following intensive pig slurry applications to natural field treatment process in Brittany (France). Environmental Pollution. 97: 119130.

LINDSAY, W.L. y NORWELL, W.A. 1969. Development of a DTPA micronutrient soil test. Agron. Abstr., 84 p.

MAPYA 1998. Métodos oficiales de análisis en la Union Europea. Diario Oficial de las Comunidades Europeas. Tomo 1. Secretaría General Técnica. Ministerio de Agricultura, Pesca y Alimentación. Neografis, S.L. Madrid. 495 p.

MAPYA. 2003. www.mapya.es

MADRID, A., MADRID, R. y VICENTE, J.M. 1996. Fertilizantes. AMV Ediciones y Mundi-prensa, $436 \mathrm{p}$.

MARTINEZ, J. y HAO, X. 1996. A field treatment plant for pig slurry. Wat. Sci. Tech. 34: 87-92

MARTINEZ, J. y PEU, P. 2000. Nutrient fluxes from a soil treatment process for pig slurry. Soil Use and Management. 16: 100-107.

MURCIA, A.J. 2003a. Principales riesgos derivados de la aplicación de purines en el Valle del Guadalentin, Lorca. Proyecto fin de carrera, Escuela Técnica Superior de Ingeniería Agronómica. Universidad Politécnica de Cartagena.

MURCIA, J.F. 2003b. Utilización del purín para fertilización en cultivo de bróculi y su comparación con la fertilización química convencional. Proyecto fin de carrera, Escuela Técnica Superior de Ingeniería Agronómica. Universidad Politécnica de Cartagena.
PAPINI, R., SPALLACCI, P y PRIMAVERA, F. 1994. Forme di fosforo in diversi suoli trattati con liquami suini e loro evoluzione nel tempo. Agrichimica. Vol. XXXVIII: 5-6.

PEECH, M. 1965. Hidrogen-ion activity. In C. A. Black (ed.). Methods of Soil Analysis. American Society of Agronomy. Madison, Wisconsin, U.S.A. 2, 914-916.

PICCININI, S. y BORTONE, G. 1991. The fertilizer value of agriculture manure: simple rapid methods of assessment. J. Agric. Engng. Res. 49: 197-208.

PLAZA, C. 2002. Aprovechamiento agrícola del purín de cerdo en agroecosistemas semiáridos: efectos sobre suelos y plantas. Tesis Doctoral. Universidad Autónoma de Madrid.

PRATT, M. 1965. Potassium and sodium. In C. A. Black (ed.). Methods of Soil Analysis American Society of Agronomy. Madison, Wisconsin, USA. 2, 1022-1030.

REGLAMENTO (CE) No 563/2002 de la Comisión de 2 de abril de 2002. Por el que se modifica el Reglamento (CE) no 466/2001 por el que se fija el contenido máximo de determinados contaminantes en los productos alimenticios.

ROCHETTE, P., ANGERS, D.A. y COTE, D. 2000a. Soil carbon and nitrogen dynamics following application of pig slurry for the 19th consecutive year: I. Carbon dioxide fluxes and microbial biomass carbon. Soil Science Society of America Journal. 64: 13891395.

ROCHETTE, P., BOCHOVE, E., PREVOST, D., ANGERS, D., COTE, D. Y BERTRAND, N. 2000b. Soil carbon and nitrogen dynamics following application of pig slurry for the 19th consecutive year: II. Nitrous oxide fluxes and mineral nitrogen. Soil Science Society of America Journal. 64: 1396-1403.

SÁNCHEZ, M. 2001. Utilización agrícola del estiércol licuado de ganado porcino: método rápido de determinación del valor fertilizante. Establecimiento de bases para el diseño de un óptimo plan de fertilización. Tesis Doctoral. Universidad de Valladolid.

SCHRÖDER, J. 2005. Revisiting the agronomic benefits of manure: a correct assessment and exploitation of its fertilizer value spares the environmental. Bioresource Technology. 96: 253-291. 
SCHRÖDER, J.J., BANNINK, A. Y KOHN, R. 2004. Improving the efficiency of nutrients use in cattle operations. En: Pfeffer, E., Hristov, A.N. (Eds), Nitrogen and Phosphorus Nutrition of Cattle. CABI, Wallingford, UK, in press.

SEYBOLD, C. A., MAUSBACH, M. J., KARLEN, D. L. y ROGERS, H.H. 1998. Quantification of soil quality. In: Lal, R., Kimble, J. M., Follet, R. F., Stewart, B. A. (Eds). Soil processes and the carbon cycle. CRC Press, BocaRaton, 387-404 p.

SIMEK, M., COOPER, J.E., PICEK, T. y SANTRUCKOVÀ, H. 2000. Denitrification in arable soils in relation to their physicochemical properties and fertilization practice. Soil Biology and Biochemistry. 32: 349-372. SØRENSEN, P y AMATO, M. 2002. Remineralisation and residual effects of $\mathrm{N}$ after application of pig slurry to soil. European Journal of Agronomy. 16: 81-95.

USDA (United States Departament of Agriculture). 2003. Keys to Soil Taxonomy. USDA (ed) 8th Eds. Washington, D.C., 326 p.
WALKER, D., CLEMENTE, R., ROIG, A y BERNAL, M.P. 2003. The effects of soil amendments on heavy metal bioavailability in two contaminated Mediterranean soils. Environmental Pollution. 122: 303-312.

WATANABE, F. S. y OLSEN, S. R. 1965. Test of ascorbic acid method for determining phosphorus in water and $\mathrm{NaHCO} 3$ extracts from soil. Soil Science Society of America Proceedings. 677-678.

WESTERNAM, P.W. y BICUDO, J.R. 2005. Management considerations for organic waste use in agriculture. Bioresource Technology. 96: 215-221.

XU, C., SHAFFER, M.J. y AL-KAISI, M. 1998. Simulting the impact of management practices on nitrous oxide emissions. Soil Science Society of America Journal. 62: 736742.

YE, Z.H., WONG, J.W., LAN, C.Y. y BAKER, A.J. 1999. Lime and pig manure as ameliorants for revegetating lead/zinc mine tailing: a greenhouse study. Bioresourse Technology. 69: 35-43. 